\title{
PERSPECTIVE
}

\section{Estimating Demand for Germline Genome Editing: An In Vitro Fertilization Clinic Perspective}

\author{
Manuel Viotti, ${ }^{1,2, *}$ Andrea R. Victor,, ${ }^{1,3}$ Darren K. Griffin, ${ }^{3}$ Jason S. Groob, ${ }^{4}$ Alan J. Brake, ${ }^{1}$ Christo G. Zouves, ${ }^{1,2}$ \\ and Frank L. Barnes ${ }^{1,2}$
}

\begin{abstract}
Germline genome editing (GGE) holds the potential to mitigate or even eliminate human heritable genetic disease, but also carries genuine risks if not appropriately regulated and performed. It also raises fears in some quarters of apocalyptic scenarios of designer babies that could radically change human reproduction. Clinical need and the availability of alternatives are key considerations in the ensuing ethical debate. Writing from the perspective of a fertility clinic, we offer a realistic projection of the demand for GGE. We lay out a framework proposing that GGE, hereditary genetic disorders, and in vitro fertilization are fundamentally entwined concepts. We note that the need for GGE to cure heritable genetic disease is typically grossly overestimated, mainly due to the underappreciated role of preimplantation genetic testing. However, we might still find applications for GGE in the correction of chromosomal abnormalities in early embryos, but techniques for that purpose do not yet exist.
\end{abstract}

\section{Introduction}

The advent of genome editing using CRISPR-based technologies is generating tremendous enthusiasm within both the medical and public domains regarding the potential to cure the $\sim 6,000$ known human genetic disorders that afflict up to $\sim 12 \%$ of the world's population. ${ }^{1,2}$

An important distinction should be drawn between germline genome editing (GGE) and somatic gene therapy. GGE could be used to modify all cells in the future organism and its offspring by targeting sperm, egg, or in most cases the fertilized zygote. The most likely scenario for the application of GGE is in preventing the transmission of inheritable genetic mutation(s), which would depend on prior knowledge of a genetic disorder. In the absence of a known disorder, a zygote is presumed healthy. However, mutations that arise de novo during embryogenesis are typically not detected until prenatal testing, during birth, or later in life, and consideration of somatic gene therapy becomes relevant to cure the individual.

In this article, writing from the perspective of the fertility clinic, we analyze the potential demand for GGE based on medical need, and explore the use of genetic testing in embryos as an alternative.

\section{In Vitro Fertilization is a Must for GGE}

In practice, GGE necessitates in vitro fertilization (IVF) laboratory techniques to provide access to the sperm, egg, and early embryos. Successful fertilization of an egg results from either insemination with several tens of thousands prepared spermatozoa or after intracytoplasmic sperm injection (ICSI) when a single sperm is injected directly into the oocyte with glass micropipettes. Both techniques result in an embryo that is typically cultured for 5-6 days in an incubator. The embryo is then either immediately placed into the patient by intrauterine transfer or, more commonly, frozen until future use when the recipient has had additional time to recover from the egg retrieval procedure. The embryo can subsequently be thawed and transferred, hopefully resulting in successful implantation.

The best-practice process of an IVF cycle under current international standards ${ }^{3}$ inherently provides direct access to germ cells and embryo to deliver the CRISPR-

\footnotetext{
'Zouves Fertility Center, Foster City, California.

${ }^{2}$ Zouves Foundation for Reproductive Medicine, Foster City, California.

${ }^{3}$ School of Biosciences, University of Kent, Canterbury, United Kingdom.

${ }^{4}$ Department of Mathematics and Statistics, Hunter College, New York, New York.
}

*Address correspondence to: Manuel Viotti, PhD, Zouves Fertility Center, Suite 100, 1241 East Hillsdale Boulevard, Foster City, CA 94131, E-mail: manuel@zouvesfoundation.org 
Cas genome editing machinery (Fig. 1A). In particular, the ICSI procedure provides a great avenue to codeliver sperm and genome editing molecules into the egg, to edit the genome of the resulting zygote. ${ }^{4}$

Can GGE be performed without IVF? Some hypothetical strategies for GGE have been suggested that do not involve classical IVF. For example, sperm or eggs are edited in a dish and replaced into the female reproductive system to attempt a subsequent "natural" fertilization. Alternatively, gonads are targeted directly, in hopes of modifying germ cells in their native location. In another version, germline stem cells are isolated from gonads, modified in a dish, and grafted back into ovary or testes. It is even conceivable to attempt targeting a naturally fertilized zygote by using uterine lavage to isolate it, editing the zygote in vitro, and transferring it back into the female. Either of those methods would most likely still be performed in the setting of a fertility clinic. Ultimately, the likeliest opportunity for application of GGE techniques will occur during conventional IVF. Not surprisingly, all published studies on human GGE so far have been carried out in IVF-generated zygotes. ${ }^{4-10}$

\section{Avoiding Inherited Genetic Disorders \\ with Preimplantation Genetic Testing}

The field of IVF has made tremendous advances since its inception 40 years ago, perhaps none bigger than the introduction of preimplantation genetic testing (PGT, previously known as PGD/PGS). Its goal is to identify the genetically healthy embryos in a patient's cohort with highest chances of implantation.

The role of PGT in avoiding transmission of familial genetic disorders has been greatly undervalued in scientific and public discourse surrounding GGE. Conceptually it
A

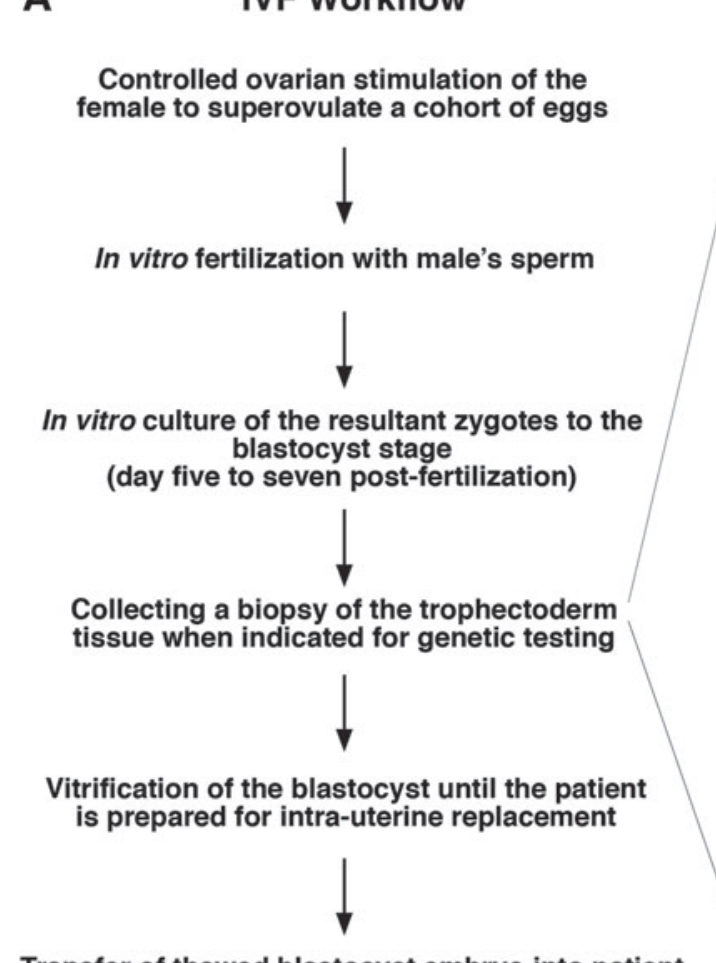

Transfer of thawed blastocyst embryo into patient

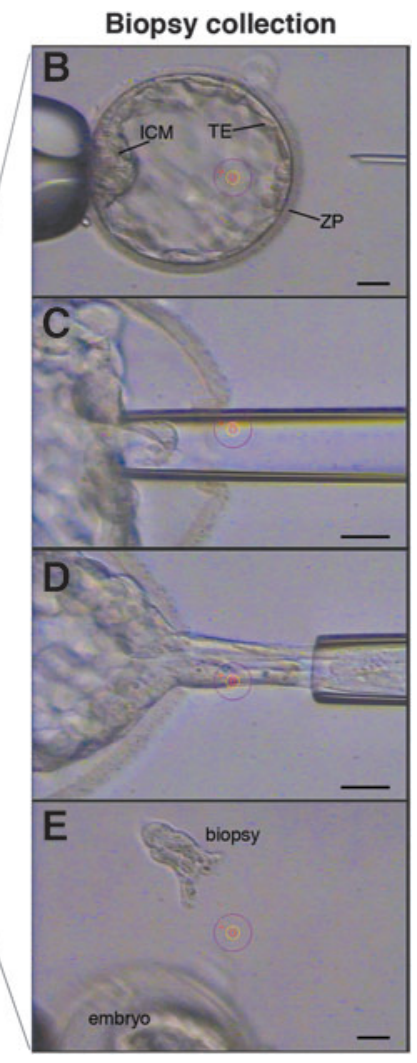

FIG. 1. Overview of the IVF process. (A) Typical workflow of an IVF cycle. (B-E) Biopsy collection for subsequent PGT. (B) Human blastocyst stage embryo with ICM at the 9 O'clock position next to a holding pipette. (C) Pulses from a laser (concentric red/yellow circles show the cross hairs) create an opening in the ZP at the 3 O'clock position, and a biopsy needle is inserted and guided to the TE tissue. (D) A clump of cells is extracted through the ZP opening by suction applied by the biopsy pipette, and laser pulses are directed at exposed cell-cell junctions.

(E) The separated 5-10 cell biopsy is collected for subsequent genetic analysis. The blastocyst is vitrified until further use. Scale bars $=50 \mu \mathrm{m}$. ICM, inner cell mass; IVF, in vitro fertilization; PGT, preimplantation genetic testing; $\mathrm{TE}$, trophectoderm; ZP, zona pellucida. 
involves isolating a cell or group of cells (the biopsy) from the developing embryo to analyze its genetic content, using it as a proxy for the entire embryo. ${ }^{3}$ Common PGT techniques currently require the isolation of a 5-10 cell biopsy at the blastocyst stage from the trophectoderm, the precursor tissue of the placenta (Fig. 1B-E). The isolated cells' DNA content is subsequently amplified and analyzed. This has proved safer to the embryo (i.e., less impact on viability) than earlier PGT methods collecting a single cell at the cleavage stage, and in addition provides more genetic material for analysis, which decreases the likelihood of erroneous or no results. ${ }^{11}$

Patients with a familial disorder can apply PGT for monogenic (-M) traits to identify embryos that have inherited the causative allele among their cohort of IVF-generated embryos and eliminate them from consid- eration for intrauterine transfer and possible implantation (Fig. 2A). The technique utilizes linkage analysis and/or direct sequencing of the causative mutation, ${ }^{12,13}$ and is in principle adaptable to any monogenic disorder, gene variants with increased disease risk, late-onset disorders, and mitochondrial DNA (mtDNA) mutations. ${ }^{11}$

In the past two decades, PGT-M has been performed in at least 100,000 IVF cycles worldwide, ${ }^{11}$ for $>400$ different single-gene disorders. ${ }^{12}$ Most recently, PGT has been designed to identify complex (polygenic) conditions, such as congenital diabetes or cardiomyopathies, in a test involving the calculation of polygenic risk scores called PGT-P. ${ }^{14}$ This test aims to provide relative risk reduction through genetic testing and identification of significant outliers. It calculates risk scores for complex conditions in embryos, not unlike services offered by direct-to-consumer genetic profiling companies. (Given

A Blastocyst Stage Embryo

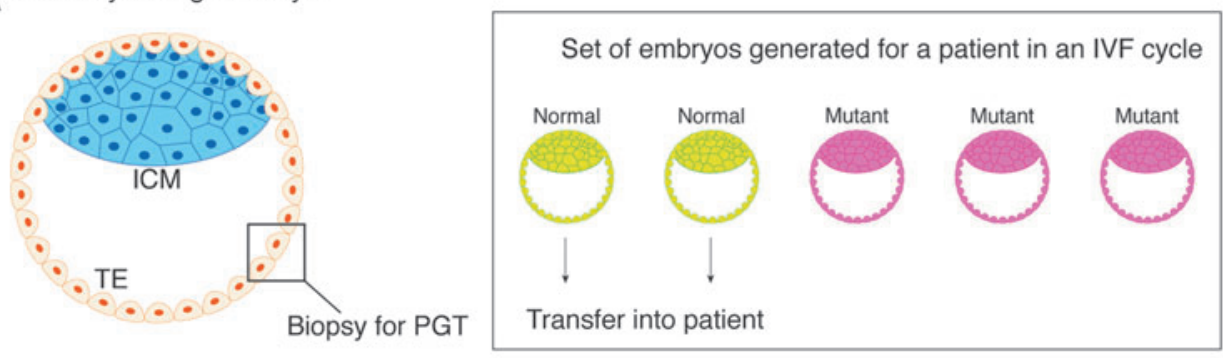

B

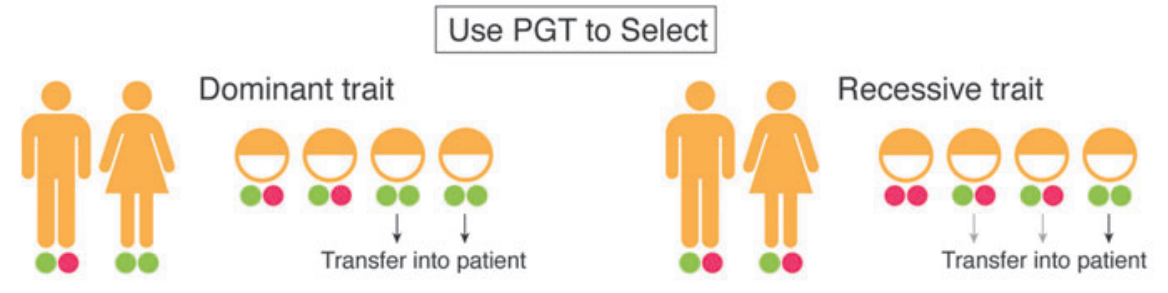

Use GGE to Correct
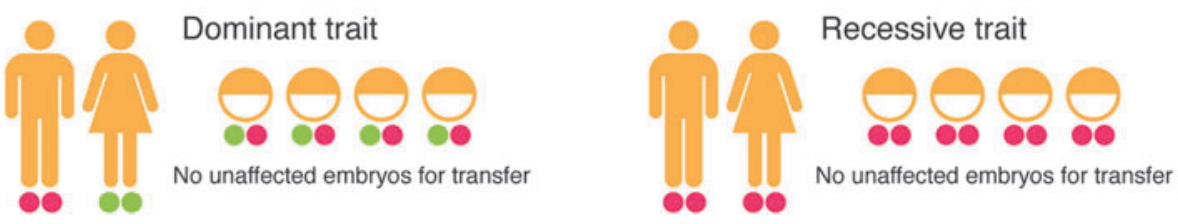

FIG. 2. Scenarios requiring PGT or GGE to prevent transmission of genetic disorders. (A) PGT requires collecting a biopsy of the embryo, which is tested for the mutation causative of the hereditary condition. The embryos in an IVF patient's cohort that do not inherit the mutation are selected for transfer and implantation. (B) PGT is the preferred method whenever embryos can be generated that are free of the mutation. When one of the parents is homozygous for a dominant disorder, or both parents are homozygous for a recessive disorder, all resulting embryos are affected, and GGE can be used to correct the mutation. GGE, germline genome editing; IVF, in vitro fertilization; PGT, preimplantation genetic testing. 
the novelty of this approach, medical societies have not yet formulated ethical statements specifically addressing polygenic testing and risk score assessment in embryos.) Other versions of PGT can be used to detect chromosomal abnormalities, from aneuploidy with PGT-A to structural rearrangements with PGT-SR.

Together, these various PGT formats are being employed in $38 \%$ of the 245,000 IVF cycles taking place each year in the United States, ${ }^{15}$ of which $10 \%$ are PGTM probing for monogenic disorders. ${ }^{16}$

\section{The Ethics of PGT Versus GGE}

Although the clinical goals of PGT and GGE to prevent the inheritance of genetic disease are similar, the ethics relevant to the two technologies is irrefutably different. In a recent position statement, the Ethics Committee of the American Society for Reproductive Medicine (ASRM) suggests that the use of PGT is ethically justified for severe congenital as well as adult-onset conditions, and furthermore endorses the use of PGT for conditions of lesser severity or penetrance if no safe, effective interventions are available. ${ }^{17}$ An important distinction between GGE and PGT is that the process of PGT does not create a new genetic trait. The constellation of genes present in the embryo selected for transfer after PGT screening could also have occurred if the couple had conceived naturally.

Unlike the practice of GGE, PGT obviously carries no risk of undesirable off- and on-target effects and no chance of introducing genetic mosaicism. However, one possible ethical argument in favor of GGE versus PGT is that, within the IVF industry, it remains standard practice to dispose of PGT embryos identified with a mutant allele or chromosomal defects. One could argue that GGE could repair and salvage these otherwise discarded embryos. However, utilizing GGE would not circumvent disposition of embryos, as it would remain unlikely that every embryo within an IVF cycle will always be transferred; often IVF patients give rise to supernumerary embryos that are routinely cryopreserved or discarded as part of IVF cycle management.

From a regulatory perspective, PGT in the United States has remained largely free from intervention by the U.S. Food and Drug Administration (FDA). Not so GGE, which clearly falls under FDA's domain as it claims "regulatory authority over genetically manipulated cells and/or their derivatives." Although somatic gene therapies have already been approved by the FDA with several trials under way, an FDA-approved GGE therapy is currently impossible. ${ }^{18} \mathrm{~A}$ renewable provision of the Consolidated Appropriation Act, initially signed into law in 2016, explicitly prohibits the FDA from reviewing appli- cations for "an exemption for investigational use of a drug or biological product...in which a human embryo is intentionally created or modified to include a heritable genetic modification."19 This moratorium in effect has been renewed every year since and its short- and longterm future are unclear. Editing the genome of a human embryo without uterine transfer for research purposes is still legal in the United States, although ineligible for public funding. ${ }^{19}$

Numerous position statements have been issued concerning GGE, ${ }^{20}$ and different ethical ramifications are currently being explored, but here we specifically consider the unmet medical need that GGE would address. When is GGE a valid medical alternative to PGT? Which is the more appropriate treatment? Does the magnitude of the medical demand for GGE justify its practice? Given the numerous technical, regulatory, and ethical concerns about the possibility of applying GGE in assisted reproduction, we should proceed very carefully. GGE would be warranted only if there is real and significant demand for the technology that could not be satisfied by other means. $^{21}$

\section{Estimation of GGE Demand for Hereditary Disorders}

Let us consider a hypothetical scenario: if a family suffers from an inherited disease, should their IVF plan be limited to PGT screening for nonaffected embryos, or are there circumstances when we should consider correcting their embryos with GGE?

In recessive monogenic disorders, each embryo conceived from two heterozygous parents will have a 1-in4 chance of inheriting two copies of the unaffected allele and a 1-in- 4 chance of being homozygous for the mutant allele. In a typical case, the unaffected embryos can be chosen for uterine transfer (Fig. 2B). Statistically, there will be some instances when every embryo of such a patient's IVF cycle might carry the mutation in question. Before considering GGE to correct the existing embryos, the couple could elect to undertake another IVF cycle with the hope of producing unaffected embryos. A typical IVF cycle will produce three to five blastocysts, but eight or more are not uncommon. In our center, the average number of blastocysts per cycle is 4.2 across all ages and indications, meaning that the likelihood of conceiving an unaffected embryo increases with subsequent multiple IVF cycles. A large study from a genetic testing laboratory reported that a mere $7.1 \%$ of all cycles with PGT-M resulted in no genetically normal embryos available for transfer. ${ }^{22}$ Those unlucky patients could subsequently do another IVF cycle and cast the die anew.

Each additional IVF cycle carries its costs, as well as a physical and emotional toll on patients. However, PGT to 
screen embryos will most likely be easier, cheaper, and safer to perform than GGE. When implementing GGE, one would likely first need to perform PGT to test which embryos inherited the mutation, and then again after GGE to confirm that the correction happened. Each intervention affects the viability of embryos, meaning a substantial increase in costs and risks. Unless methods are developed to efficiently edit all cells in a multicellular embryo, one is confined to performing the GGE intervention at the zygote stage, meaning that prior genetic testing is impossible (the process destroys the cell being analyzed). Genome editing molecules would need to be applied "blindly" into every zygote, affected and unaffected alike. Considering this and the possibility for off-target effects of GGE, we submit that hereditary conditions will continue to be handled preferentially by screening embryos with PGT over correcting them with GGE.

\section{Scenarios Favoring GGE over PGT}

Do situations exist when PGT does not work? The answer is emphatically "Yes." PGT is pointless if dealing with a recessive disorder when both parents are homozygous mutants, as all resulting embryos will be homozygous mutants as well. Only GGE would prevent transmission of the disorder. Similarly, if there is a familial autosomal dominant disorder and one or both parents are homozygous for the mutant allele, every embryo will inherit at least one copy of the mutation and be affected (Fig. 2B). But what is the frequency of those scenarios?

Previous commentaries have broached the subject, ${ }^{21,23,24}$ but a thorough analysis based on genetic epidemiology is warranted. In an effort to answer that question, we used published prevalence data for the most common genetic disorders in the United States to project how many conceptions would benefit from GGE per year on a national level (Table 1).

For common recessive disorders such as cystic fibrosis (CF), sickle cell disease (SCD), or Tay-Sachs, we calculated the chances of two homozygous mutant individuals forming a reproductive couple by random chance (see Some Caveats section for assumptions in these projections). For example, the number of people living in the United States with CF who are homozygous for CFTR mutations is estimated at 30,000, which means the prevalence in the general population is roughly 1 in $\sim 10,000$. The likelihood of two CF patients forming a couple is 1 in $\sim 10,000$ (assuming each affected individual pairs with a single mate). ${ }^{2}$ We estimate that in the United States, there is only one couple of reproductive age at any given time where both partners are homozygous for CF mutant alleles (the exact calculated value is 1.26 couples). Consid- ering the current data for the population percentage based on reproductive age, average fertility rate per woman, and range of reproductive years, we can estimate the number of conceptions each year in the United States for which GGE would be applicable. For CF, this number is negligible- $\mathrm{a}$ mere 0.065 cases per yearindicating we could anticipate that the need for GGE treatment to prevent the transmission of $\mathrm{CF}$ would occur only once every 15 years, with all other CF cases relying on PGT.

We performed calculations for other recessive genetic disorders and considered instances for which assortative mating and variable ethnical prevalence are relevant, such as SCD in the African American population and Tay-Sachs in Ashkenazi Jews. The number of projected cases is remarkably small for all of the most common single-gene recessive disorders, ${ }^{22}$ totaling fewer than a dozen clinical cases of estimated children born per year in the United States that might hypothetically benefit from GGE (Table 1).

A more complex inheritance pattern is found in $\alpha$ thalassemia, possibly the most common severe genetic disorder in the general U.S. population, due to the four alleles on two HBA genes. If one or two alleles are mutant, the phenotype is generally asymptomatic. When three alleles are mutant, the condition is known as hemoglobin $\mathrm{H}(\mathrm{HbH})$ disease and patients often suffer from anemia, hepatosplenomegaly, and jaundice. Harboring four mutant alleles causes the condition hemoglobin Bart's hydrops fetalis syndrome (BHFS), which is almost always lethal during gestation or shortly after birth. In the literature, 69 cases of BHFS have been documented that have survived past infancy, with 18 instances of survival past 10 years of age. ${ }^{25}$ The demand for GGE for $\alpha$ thalassemia is, therefore, virtually nil, as there will likely never be a couple with BHFS intending to procreate. In the case that two $\mathrm{HbH}$ patients would like to have children, one in four embryos generated would be asymptomatic carriers, one in two embryos would have $\mathrm{HbH}$, and one in four embryos would die in utero with BHFS. ${ }^{26}$ In this case, utilizing PGT to facilitate selection of an asymptomatic carrier would likely be favored over attempting GGE.

Homozygosity for severe dominant disorders is almost always embryonically lethal, making the number of such individuals virtually nonexistent in the general population. For example, the number of cases homozygous for Huntington's disease is in the dozens. ${ }^{23}$ There have been 13 recorded patients homozygous for myotonic dystrophy type $1 .{ }^{27}$ Homozygotes for mutations causing neurofibromatosis type 1 have never been documented. ${ }^{28}$ Achondroplasia, one of the most common dominant 


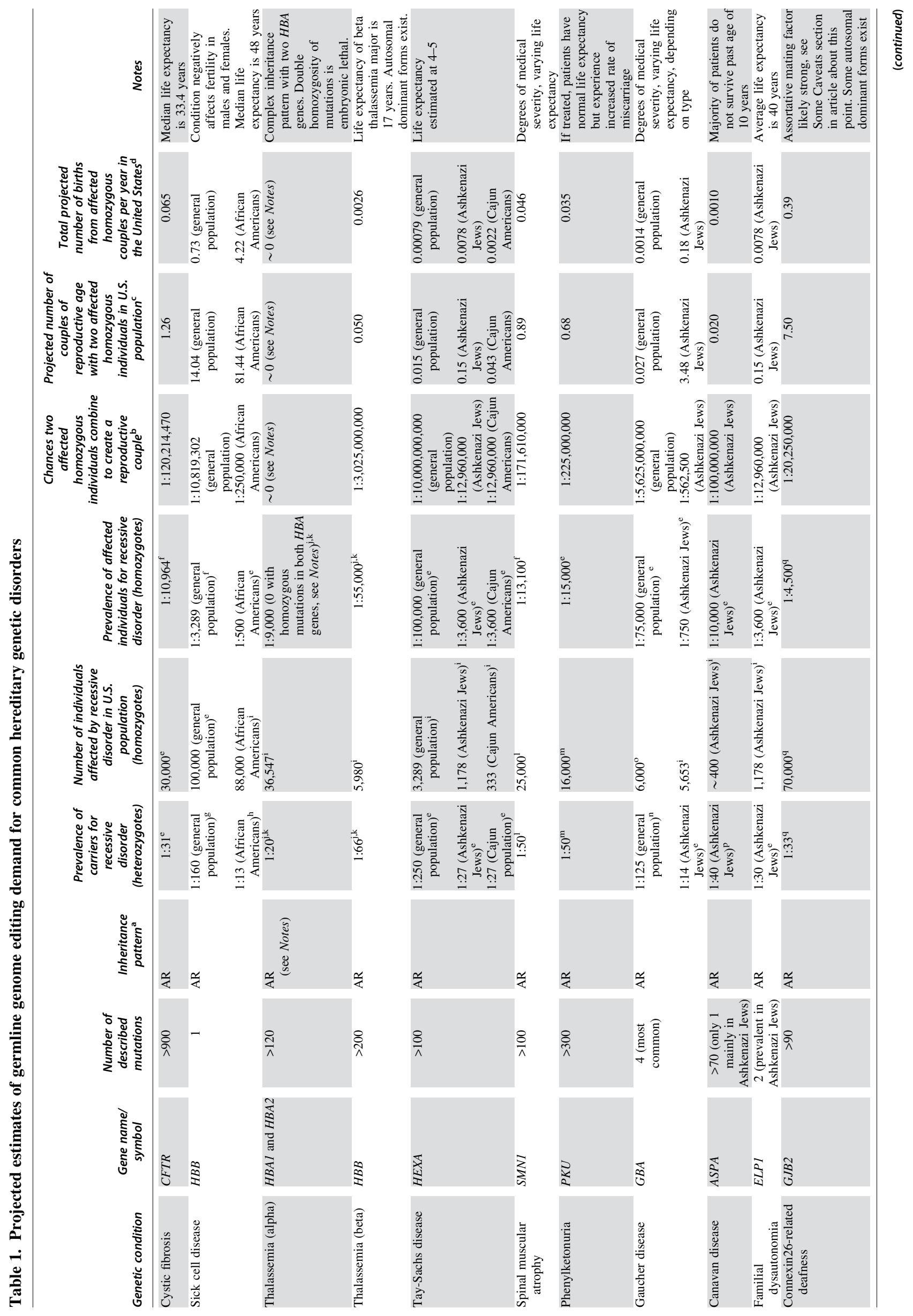




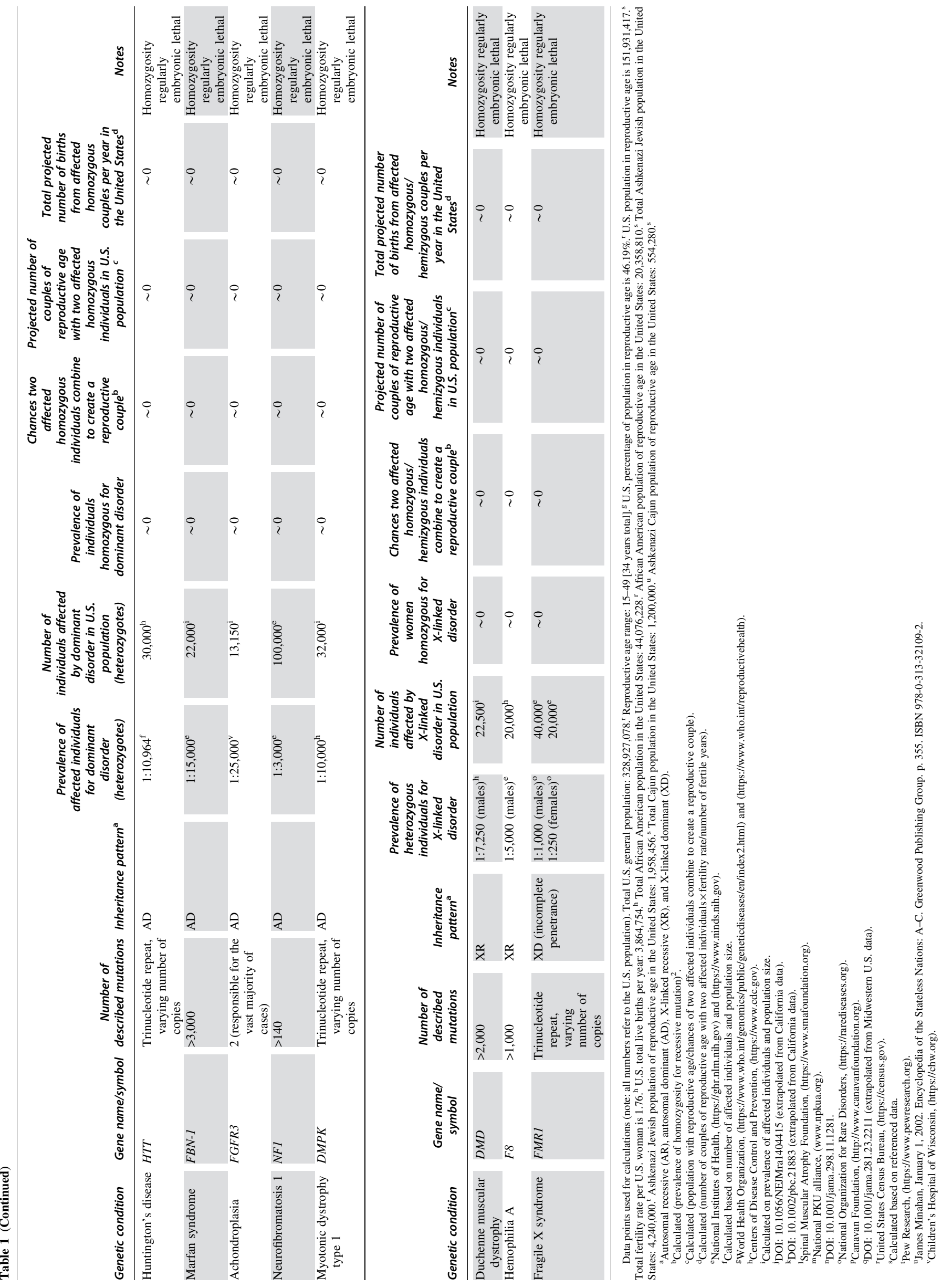


disorders, is caused by a mutation in FGFR3. When heterozygous, it causes dwarfism, but is considered uniformly fatal when homozygous, and only a handful of cases are reported to have survived into early childhood. ${ }^{29}$ Therefore, the projected annual national demand of GGE for dominant disorders approaches zero, as PGT is applicable to identify nonaffected embryos in virtually all cases.

PGT is also appropriate for cases of X-linked disorders. Female carriers of a recessive X-linked disorder have a one in four chance with each pregnancy to have a carrier daughter, a one in four chance to have a noncarrier daughter, a one in four chance to have a son affected with the disease, and a one in four chance to have an unaffected son. PGT can identify affected male embryos. In cases where the mother is homozygous for the mutation, all the male offspring would inherit a mutant copy of X. Here again, just with autosomal dominant disorders, the prevalence of homozygosity for X-linked disorders is remarkably low. ${ }^{30}$ For example, Duchenne muscular dystrophy has never been identified as homozygous. ${ }^{31}$ Hemophilia A, which affects 1:5,000 male births, has only ever been documented as homozygous a few times. ${ }^{32-35}$ Fragile $\mathrm{X}$ syndrome, considered a dominant $\mathrm{X}$-linked disorder, is characterized by amplification of a trinucleotide repeat, and has only been described as homozygous in the premutation form, not the fully amplified allele. ${ }^{36}$

One clinical situation that would benefit from genome editing is a male affected by a dominant X-linked disorder who wishes to conceive children. His male embryos will be disease free by default. But a daughter would undoubtedly inherit his affected X chromosome. Such a circumstance prompts a different ethical discussion revolving around gender selection, and specifically the justification for wanting a female child that must be genetically altered rather than choosing a healthy male. Here again, due to PGT, the number of cases who would require GGE is extremely small.

What about less severe hereditary conditions? It has been suggested that couples with congenital hearing loss, which affects 1 in 500 individuals, might consider GGE to have a hearing-enabled child. ${ }^{23}$ Deafness is genetically heterogeneous with $>1,000$ mutations identified in $>90$ genes, but recessively inherited single-gene mutations in GJB2 are responsible for over half of all cases. ${ }^{37}$ Historically, deafness has been a prime example for assortative mating. In the 1970 s, $80-90 \%$ of people with profound deafness in the United States married another deaf person. ${ }^{38}$ It is estimated that 1 person in 4,500 is homozygous mutant for GJB2. ${ }^{39}$ Assuming that all GJB2-mutant homozygotes of reproductive age intermarried and repro- duced at general rates, there could be a potential 837 conceptions nationally per year that would benefit from editing $G J B 2$, which is a sizable number.

However, the fact that deafness is a treatable condition invalidates this projection. The introduction of cochlearimplant technology has profoundly altered the mating structure of the deaf population. ${ }^{40}$ By facilitating oral communication and educational mainstreaming, practically all deaf children of hearing parents are redirected into the hearing mating pool. ${ }^{40}$ This is an example in which treatment might obviate the need for GGE and its associated risks. We predict that GGE will find little demand in less severe or treatable genetic conditions, considering medical necessity and alternative interventions. This was also one of the main criticisms of the only alleged case of human GGE resulting in births reported to date, where CCR 5 was mutated to diminish chances of possible HIV infection-proven strategies already exist for the prevention of perinatal transmission of HIV. ${ }^{41}$

GGE might be the only strategy for IVF patients who carry a genetic condition but have conceived only affected embryos and are precluded from initiating additional IVF cycles because of advancing age, disease, or cost. Of the annual $\sim 100,000 \mathrm{IVF}$ cycles in the United States accompanied by PGT, ${ }^{15}$ the fraction using PGT$\mathrm{M}$ for single-gene disorders is estimated at $10 \% .{ }^{16}$ Of those, $7.1 \%$ are estimated not to generate any normal embryos, ${ }^{22}$ resulting in $\sim 7,100$ cycles. Judging from observations in our IVF center, we estimate $<1 \%$ of patients not being able to perform a subsequent IVF cycle for PGT-M when desired. Hence, we project there to be at most 71 cases per year in the United States who would benefit from GGE for this particular scenario, if all patients elected to pursue that procedure.

It should be noted that the unicellular zygote cannot be tested for a genetic disorder without destroying it. It is, therefore, not feasible to screen zygotes for a mutant allele and then attempt to edit that allele. GGE at the zygote stage makes sense only when there is previous knowledge of that zygote's genetic makeup, as when the complement is deduced from homozygosity for the mutant allele in one or both parents. One exception is with maternal heterozygosity, as one can attempt deducing the status of the zygote by analyzing polar bodies, which are the products of meiosis. Although historically there has been some limited success, this practice produces notoriously high amounts of false positives and negatives. The state of heterozygosity in the mother would permit conventional PGT screening for embryos not having inherited the allele, precluding the need for GGE. Therefore, even when there would be demand for GGE, the gene editing machinery would only be administered later in 
development, likely at the blastocyst stage when most PGT takes place and affected embryos are identified. However, the expertise does not yet exist to deliver CRISPR-Cas to all 64-128 cells of a blastocyst and prevent any incidence of the potential medical risks associated with resultant mosaicism.

Together, our analysis suggests that the true clinical demand for GGE for single-gene hereditary disorders is exceedingly small. We estimate that GGE could at most benefit 100 births per year in the United States, when all eligible patients opted for the procedure.

\section{Use of GGE in mtDNA Disease}

mtDNA disease is relatively common, with an estimated prevalence of 1 in 5,000, ${ }^{42}$ and causes severe and debilitating genetic disorders such as Leigh disease, KearnsSayre syndrome, or Mitochondrial encephalomyopathy, lactic acidosis, and stroke-like episodes (MELAS). Most mtDNA mutations occur de novo, and maternal transmission of mtDNA disease is rare. There are an estimated $\sim 800$ pregnancies per year in the United States at risk of maternal transfer of mutant mtDNA. ${ }^{43}$ Owing to the multicopy nature of mtDNA, mutant mtDNA often exists in a mix with normal mtDNA in a cell, a state known as heteroplasmy. In most cases, PGT-M can be designed to identify and quantify mutations in mtDNA to deselect embryos with high mutation load. The circumstances in which PGT-M is not useful are those in which there is homoplasmy of mutant mtDNA, or when the patient is only capable of producing heteroplasmic embryos with high mutation load. In those instances, GGE might reduce the mutation load by correcting mutations in as many mtDNA copies as possible. Even with such a treatment, GGE would need to be proven more effective and safer than the alternative treatment of mitochondrial replacement therapy. ${ }^{44}$ Taking these factors into account, we conclude that the U.S. annual demand for GGE for mtDNA disorders might be in the low dozens.

\section{Can GGE Cure Embryonic Aneuploidy and Other Chromosomal Abnormalities?}

PGT-A and PGT-SR, which test embryos for chromosomal abnormalities, are now performed in $>30 \%$ of IVF cycles in the United States and growing. ${ }^{15,16}$ Some of those instances are for familial conditions, for which there is a risk of inheriting a chromosomal abnormality. However, PGT-A/PGT-SR is mostly utilized as a screen, even when both parents are chromosomally normal. Chromosomal abnormalities are common de novo events in naturally conceived embryos as well as in those generated by IVF, ${ }^{11}$ and embryos that carry chromosomal abnormalities are developmentally compromised and often fail to implant. When implantation does occur, the pregnancy often results in miscarriage. In the few instances wherein they make it to birth, the offspring is bound to harbor chromosomal conditions ranging from whole chromosome monosomies or trisomies (e.g., Down, Turner, or Edwards syndromes), subchromosomal segmental deletions or duplications (e.g., Cri-du-Chat, Angelman, or Prader-Willi syndromes), or rearrangements that can lead to a range of conditions. PGT-A/PGT-SR facilitates the deselection of such embryos before intrauterine transfer, and numerous randomized controlled trials and observational studies have reported a significant increase in clinical success rates when embryos classified as normal/euploid are used in comparison with blindly selecting embryos for transfer into the patient. ${ }^{45-47}$

Importantly, the observed rates of embryo aneuploidy increase with advancing maternal age due to errors of meiosis during oocyte maturation, a process that becomes more error prone over time. ${ }^{48}$ On average, rates of aneuploidy increase from $30 \%$ of IVF embryos in women in their early 30 s to nearly $90 \%$ for women aged 44 years and above. ${ }^{49}$ This correlation between maternal age and proportion of aneuploid embryos means that classical Mendelian calculations do not apply. In contrast with hereditary single-gene disorders wherein there is a high likelihood of conceiving a genetically normal embryo at each IVF cycle, a patient with advanced maternal age has an elevated risk of producing only aneuploid embryos even after multiple IVF cycles. In addition, a large study analyzing $>15,000$ IVF embryos has shown that there is even a subset of younger patients also only capable of generating aneuploid embryos, ${ }^{49}$ possibly due to faulty cell cycle mechanisms in those patients. When a couple is only able to generate chromosomally abnormal embryos, even after repeated IVF cycles, the result would often be their abandonment of infertility treatment altogether.

Could GGE correct chromosomal abnormalities in embryos, such as erasure of the additional chromosome present in trisomic embryos? Even though this technology does not yet exist, intriguing new data have emerged with proof-of-concept experiments targeting and deleting of entire chromosomes. ${ }^{50,51}$ What is the potential clinical demand for that procedure? Internal data from 1,769 IVF cycles at our IVF center indicate that $\sim 20 \%$ of initial cycles produce only aneuploid embryos, and a subsequent cycle for those patients has $\sim 60 \%$ chance of containing only aneuploid embryos. We also observe that $\sim 100 \%$ of patients who produce only aneuploids after three cycles discontinue their IVF treatment. Extrapolating those figures to the $\sim 245,000$ IVF cycles currently performed annually in the United States, ${ }^{15}$ we estimate that $\sim 17,600$ patients would benefit from GGE of aneuploid embryos. 
A different application for this kind of GGE is for individuals with Robertsonian translocations. Such individuals are typically healthy except for their increased chances of generating embryos with aneuploidies. Robertsonian translocation 21q; $21 \mathrm{q}$ is particularly problematic, since $100 \%$ of resulting embryos have trisomy 21 and, therefore, Down's syndrome if carried to term. ${ }^{52}$ Since PGT-A for screening is not beneficial, this situation would meet the requirement for unmet medical need.

It is to be noted that this practice would not be considered GGE in the classical sense, because the target is not the zygote but a 64-128-cell blastocyst. Unless techniques are developed to target every cell in the blastocyst, the result will inevitably be mosaicism. Interestingly, recent data have shown that blastocysts classified by PGTA as chromosomally mosaic (harboring a mix of euploid and aneuploid cells) can result in implantation and healthy pregnancies, although with significantly lower success rates than blastocysts classified as euploid. ${ }^{53-55}$ To date, $\sim 300$ babies born from transferred mosaic embryos have not presented medical complications of any kind. ${ }^{53-55}$ Evidence from mouse and human embryology indicates that in a mosaic embryo euploid cells dilute out aneuploid cells as the latter preferentially die off or divide at slower rates during development. ${ }^{55,56}$

Deleting an extra chromosome when trisomy is present would typically imply the creation of uniparental disomy, since the GGE machinery would need to target the chromosome that is dissimilar and contains differentiating DNA sequences. The implication is that the remaining two chromosomes would stem from the same parent, potentially creating epigenetic consequences with medical manifestations. Testing parental genomes, polar bodies, and deep sequencing of the TE biopsy in PGT-A might help identify exploitable differentiating regions between duplicated chromosomes. More research is needed in this field, and careful consideration will have to be given to what regions/chromosomes can be targeted to rid an embryo of aneuploidy.

In summary, the prospect of GGE for chromosomal correction could have a tremendous impact on IVF patients, although many technical advances and much more research are needed.

\section{Some Caveats}

We must note some significant assumptions within our calculations. Several of the genetic disorders described here shorten life expectancies and alter life conditions to the point of skewing statistics regarding fertility for these populations. For example, the median age of survival for SCD is 48 years, for $\mathrm{CF}$ it is 33.4 years, and for Tay-Sachs it is $4-5$ years. The likelihood of two af- fected individuals meeting is not entirely up to chance (as the simple calculations suggest). Having the same disorder could make it more likely for two patients to come together to form a couple (assortative mating); individuals with a particular condition are more likely to meet others with the same condition through societies, associations, and help groups. In contrast, certain genetic disorders affect the immune system and increase risk of infection, making it risky to interact with other patients of the same condition. For example, CF patients are recommended to maintain a minimal distance of $2 \mathrm{~m}$ from each other at all times due to the risk of bacterial cross-infection. ${ }^{57}$

The incidence of genetic disorders is also altered by sociocultural and ethnicity factors such as consanguinity, endogamy, and high prevalence in certain groups, such as Tay-Sachs or SCD. Penetrance and variable expressivity have been disregarded for simplicity. Mutations causing disorders with treatable symptoms such as hemochromatosis, or mutations that increase disease risk such as $B R C A 1 / 2$, were generally excluded from this analysis as testing for those conditions is not common or remains controversial; performing GGE for those factors is even more unlikely. Importantly, our calculations using published prevalence data assume that all cases of a genetic disorder are inherited, although a substantial percentage of de novo mutations cases do occur for particular types of inherited disorders. Taken together, these assumptions suggest that our projections are likely inflated, and the actual demand of GGE targeting heritable genetic disorders is probably even lower than the figures already presented.

\section{Nonmedical Enhancements}

We have not considered the hypothetical demand for GGE to introduce genome edits of a nonmedical nature for enhancement. The prospect of "designer babies" whose DNA is sculpted by GGE to control features such as intelligence, height, and longevity would require the development of safe methods to edit dozens, hundreds, or maybe thousands of loci in the genome. Complex traits are multifactorial and the full set of involved genes remains unknown. PGT-P could potentially be used to screen embryos for such enhancements, for example, ranking the embryos in a patient's cohort for predicted height. Aside from the obvious ethical concerns, we believe the actual demand for such nonmedical screening will be negligible. IVF laboratories have had the capability of using PGT to select for traits such as eye color, for example, but this has not become common practice. ${ }^{16}$

\section{Conclusions}

Our calculations should not be considered exact predictions of the potential demand for GGE. They are intended 
to provide some calibration of expectations. GGE is inevitably entwined with IVF, a medical field that has long dealt with screening for familial genetic disorders using PGT. Couples in which one or both partners are affected by a genetic disorder would necessarily need to undergo IVF whether the strategy to prevent propagation of the causative allele involved GGE or PGT. Given the choice, we believe that PGT will always be preferred.

Our projections show there are extremely few instances for which GGE will be the only option, such as when both parents are homozygotes for a recessive condition or at least one parent is homozygous for a dominant condition. We do not intend to downplay the importance of those rare cases and patients. Nonetheless, the actual magnitude of the demand for GGE must be part of the conversation when debating ethical and legal frameworks. However, we suggest that aneuploidy might be a much larger issue than previously considered, as we predict there will be many potential beneficiaries of chromosomal editing in embryos if safe technologies for that purpose can be developed.

\section{Authors' Contributions}

M.V. conceptualized the subject, wrote the article, and prepared the tables and figures. A.R.V. coconceptualized the subject and edited the article. D.K.G. edited the article. J.S.G. oversaw the mathematical calculations and edited the article. A.J.B. edited the article. C.G.Z. coconceptualized the subject and edited the article. F.L.B. coconceptualized the subject and edited the article. All coauthors have reviewed and approved of the article before submission.

\section{Author Disclosure Statement}

No competing financial interests exist.

\section{Funding Information}

Funding for this study was provided by the Zouves Foundation for Reproductive Medicine.

\section{References}

1. Online Mendelian Inheritance in Man (OMIM). McKusick-Nathans Institute of Genetic Medicine, Johns Hopkins University (Baltimore, MD). Available online at https://omim.org (last accessed June 3, 2019).

2. Assembly of Life Sciences (U.S.). Committee on the Biological Effects of lonizing Radiations. The Effects on populations of exposure to low levels of ionizing radiation, 1980. Washington, DC: National Academy Press. 1980.

3. Maxwell SM, Grifo JA. Should every embryo undergo preimplantation genetic testing for aneuploidy? A review of the modern approach to in vitro fertilization. Best Pract Res Clin Obstet Gynaecol. 2018;53:38-47. DOI: 10.1016/j.bpobgyn.2018.07.005.

4. Ma H, Marti-Gutierrez N, Park SW, et al. Correction of a pathogenic gene mutation in human embryos. Nature. 2017;548:413-419. DOI: 10.1038/ nature23305.

5. Fogarty NME, McCarthy A, Snijders KE, et al. Genome editing reveals a role for OCT4 in human embryogenesis. Nature. 2017;550:67-73. DOI: $10.1038 /$ nature24033.
6. Liang $P, X u Y$, Zhang $X$, et al. CRISPR/Cas9-mediated gene editing in human tripronuclear zygotes. Protein Cell. 2015;6:363-372. DOI: 10.1007/s13238-015-0153-5.

7. Zeng Y, Li J, Li G, et al. Correction of the Marfan syndrome pathogenic FBN1 mutation by base editing in human cells and heterozygous embryos. Mol Ther. 2018;26:2631-2637. DOI: 10.1016/j.ymthe.2018.08.007.

8. Liang P, Ding C, Sun H, et al. Correction of beta-thalassemia mutant by base editor in human embryos. Protein Cell. 2017;8:811-822. DOI: 10.1007/s13238-017-0475-6.

9. Tang L, Zeng Y, Du H, et al. CRISPR/Cas9-mediated gene editing in human zygotes using Cas9 protein. Mol Genet Genomics. 2017;292:525-533. DOI: 10.1007/s00438-017-1299-z.

10. Kang $X$, He W, Huang $Y$, et al. Introducing precise genetic modifications into human 3PN embryos by CRISPR/Cas-mediated genome editing. J Assist Reprod Genet. 2016;33:581-588. DOI: 10.1007/s10815-016-0710-8.

11. Griffin DK, Ogur C. Chromosomal analysis in IVF: Just how useful is it? Reproduction. 2018;156:F29-F50. DOI: 10.1530/REP-17-0683.

12. Kuliev A, Rechitsky S. Preimplantation genetic testing: Current challenges and future prospects. Expert Rev Mol Diagn. 2017;17:1071-1088. DOI: 10.1080/14737159.2017.1394186.

13. Grace J, El-Toukhy T, Scriven P, et al. Three hundred and thirty cycles of preimplantation genetic diagnosis for serious genetic disease: Clinical considerations affecting outcome. BJOG. 2006;113:1393-1401. DOI: 10.1111/j.1471-0528.2006.01143.x.

14. Treff NR, Zimmerman R, Bechor $E$, et al. Validation of concurrent preimplantation genetic testing for polygenic and monogenic disorders, structural rearrangements, and whole and segmental chromosome aneuploidy with a single universal platform. Eur J Med Genet. 2019;62:103647. DOI: 10.1016/j.ejmg.2019.04.004.

15. Society for Assisted Reproductive Technology (SART). Available online at htpps://www.sart.org (last accessed June 3, 2019).

16. Munne S. Status of preimplantation genetic testing and embryo selection. Reprod Biomed Online. 2018;37:393-396. DOI: 10.1016/ j.rbmo.2018.08.001.

17. Ethics Committee of the American Society for Reproductive Medicine. Electronic address Aao, Ethics Committee of the American Society for Reproductive $M$. Use of preimplantation genetic testing for monogenic defects (PGT-M) for adult-onset conditions: An Ethics Committee opinion. Fertil Steril. 2018;109:989-992. DOI: 10.1016/j.fertnstert.2018.04.003.

18. Gleicher N. Expected advances in human fertility treatments and their likely translational consequences. J Transl Med. 2018;16:149. DOI: 10.1186/s12967-018-1525-4.

19. Cohen IG, Adashi EY. Science and regulation. The FDA is prohibited from going germline. Science. 2016;353:545-546. DOI: 10.1126/science.aag2960.

20. Brokowski C. Do CRISPR germline ethics statements cut it? CRISPR J. 2018;1:115-125. DOI: 10.1089/crispr.2017.0024.

21. Dance A. Better beings? Nat Biotechnol. 2017;35:1006-1011. DOI: 10.1038/ nbt.3998.

22. Gutierrez-Mateo C, Sanchez-Garcia JF, Fischer J, et al. Preimplantation genetic diagnosis of single-gene disorders: Experience with more than 200 cycles conducted by a reference laboratory in the United States. Fertil Steril. 2009;92:1544-1556. DOI: 10.1016/j.fertnstert.2008.08.111.

23. Lander ES. Brave new genome. N Engl J Med. 2015;373:5-8. DOI: 10.1056/ NEJMp1506446.

24. Araki $M$, Ishii T. International regulatory landscape and integration of corrective genome editing into in vitro fertilization. Reprod Biol Endocrinol. 2014;12:108. DOI: 10.1186/1477-7827-12-108.

25. Songdej D, Babbs $C$, Higgs DR, et al. An international registry of survivors with Hb Bart's hydrops fetalis syndrome. Blood. 2017;129:1251-1259. DOI: 10.1182/blood-2016-08-697110.

26. Piel FB, Weatherall DJ. The alpha-thalassemias. N Engl J Med. 2014;371:1908-1916. DOI: 10.1056/NEJMra1404415.

27. Carroll JM, Quaid KA, Stone K, et al. Two is better than one: A case of homozygous myotonic dystrophy type 1 . Am J Med Genet $A$. 2013;161A:1763-1767. DOI: 10.1002/ajmg.a.35967.

28. Jouhilahti EM, Peltonen S, Heape AM, et al. The pathoetiology of neurofibromatosis 1. Am J Pathol. 2011;178:1932-1939. DOI: 10.1016/ j.ajpath.2010.12.056. 
29. Pauli RM, Conroy MM, Langer LO, Jr., et al. Homozygous achondroplasia with survival beyond infancy. Am J Med Genet. 1983;16:459-473. DOI: 10.1002/ajmg.1320160404.

30. Germain DP. General aspects of X-linked diseases In: Fabry Disease: Perspectives from 5 Years of FOS. (Mehta A, Beck M, Sunder-Plassmann G; eds). Oxford, United Kingdom: Oxford Pharmagenesis, 2006;63-68.

31. Fujii K, Minami N, Hayashi Y, et al. Homozygous female Becker muscular dystrophy. Am J Med Genet A. 2009;149A:1052-1055. DOI: 10.1002/ ajmg.a.32808.

32. Nair PS, Shetty S, Ghosh K. A homozygous female hemophilia A. Indian J Hum Genet. 2012;18:134-136. DOI: 10.4103/0971-6866.96685.

33. Morita $H$, Kagami M, Ebata $Y$, et al. The occurrence of homozygous hemophilia in the female. Acta Haematol. 1971;45:112-119. DOI: 10.1159/ 000208614.

34. Pola V, Svojitka J. [Classic hemophilia in women]. Folia Haematol Int Mag Klin Morphol Blutforsch. 1957;75:43-51.

35. Sie $P$, Caranobe $C$, Benalioua $M$, et al. Homozygous hemophilia $A$ in a female. Thromb Haemost. 1985;54:728.

36. Mazzocco MM, Holden JJ. Neuropsychological profiles of three sisters homozygous for the fragile X premutation. Am J Med Genet. 1996;64:323-328. DOI: 10.1002/(SICI)1096-8628(19960809)64:2 $<323::$ AID-AJMG18>3.0.CO;2-H

37. Martinez AD, Acuna R, Figueroa V, et al. Gap-junction channels dysfunction in deafness and hearing loss. Antioxid Redox Signal. 2009;11:309322. DOI: 10.1089 /ars.2008.2138

38. Schein JD, Delk MT, National Association of the Deaf. The deaf population of the United States. Silver Spring, MD: National Association of the Deaf. 1974.

39. Green GE, Scott DA, McDonald JM, et al. Carrier rates in the midwestern United States for GJB2 mutations causing inherited deafness. JAMA. 1999;281:2211-2216. DOI: 10.1001/jama.281.23.2211.

40. Arnos KS, Welch KO, Tekin M, et al. A comparative analysis of the genetic epidemiology of deafness in the United States in two sets of pedigrees collected more than a century apart. Am J Hum Genet. 2008;83:200-207. DOI: 10.1016/j.ajhg.2008.07.001.

41. Zhang $L$, Zhong $P$, Zhai $X$, et al. Open letter from Chinese HIV professionals on human genome editing. Lancet. 2019;393:26-27. DOI: 10.1016/S0140-6736(18)33082-4.

42. Gorman GS, Schaefer AM, Ng Y, et al. Prevalence of nuclear and mitochondrial DNA mutations related to adult mitochondrial disease. Ann Neurol. 2015;77:753-759. DOI: 10.1002/ana.24362.

43. Gorman GS, Grady JP, Turnbull DM. Mitochondrial donation-How many women could benefit? N Engl J Med. 2015;372:885-887. DOI: 10.1056/ NEJMc1500960.

44. Zhang J, Liu H, Luo S, et al. Live birth derived from oocyte spindle transfer to prevent mitochondrial disease. Reprod Biomed Online. 2017;34:361368. DOI: 10.1016/j.rbmo.2017.01.013.
45. Scott RT, Jr., Upham KM, Forman EJ, et al. Blastocyst biopsy with comprehensive chromosome screening and fresh embryo transfer significantly increases in vitro fertilization implantation and delivery rates: $\mathrm{A}$ randomized controlled trial. Fertil Steril. 2013;100:697-703. DOI: 10.1016/j.fertnstert.2013.04.035.

46. Yang Z, Liu J, Collins GS, et al. Selection of single blastocysts for fresh transfer via standard morphology assessment alone and with array $C G H$ for good prognosis IVF patients: Results from a randomized pilot study. Mol Cytogenet. 2012;5:24. DOI: 10.1186/1755-8166-5-24.

47. Dahdouh EM, Balayla J, Garcia-Velasco JA. Comprehensive chromosome screening improves embryo selection: A meta-analysis. Fertil Steril. 2015;104:1503-1512. DOI: 10.1016/j.fertnstert.2015.08.038.

48. Hassold T, Hunt P. To err (meiotically) is human: The genesis of human aneuploidy. Nat Rev Genet. 2001;2:280-291. DOI: 10.1038/ 35066065.

49. Franasiak JM, Forman EJ, Hong $\mathrm{KH}$, et al. The nature of aneuploidy with increasing age of the female partner: A review of 15,169 consecutive trophectoderm biopsies evaluated with comprehensive chromosomal screening. Fertil Steril. 2014;101:656.e651-663.e651. DOI: 10.1016/ j.fertnstert.2013.11.004.

50. Adikusuma F, Williams N, Grutzner F, et al. Targeted deletion of an entire chromosome using CRISPR/Cas9. Mol Ther. 2017;25:1736-1738. DOI: 10.1016/j.ymthe.2017.05.021.

51. Zuo E, Huo X, Yao X, et al. CRISPR/Cas9-mediated targeted chromosome elimination. Genome Biol. 2017;18:224. DOI: 10.1186/s13059-0171354-4.

52. Kolgeci $\mathrm{S}$, Azemi $\mathrm{M}$, Ahmeti $\mathrm{H}$, et al. Recurrent abortions and down syndrome resulting from Robertsonian translocation 21q; 21q. Med Arch. 2012;66:350-352.

53. Munne S, Blazek J, Large M, et al. Detailed investigation into the cytogenetic constitution and pregnancy outcome of replacing mosaic blastocysts detected with the use of high-resolution next-generation sequencing. Fertil Steril. 2017;108:62.e68-71.e68. DOI: 10.1016/j.fertnstert.2017.05.002.

54. Spinella F, Fiorentino F, Biricik A, et al. Extent of chromosomal mosaicism influences the clinical outcome of in vitro fertilization treatments. Fertil Steril. 2018;109:77-83. DOI: 10.1016/j.fertnstert.2017.09.025.

55. Victor AR, Tyndall JC, Brake AJ, et al. One hundred mosaic embryos transferred prospectively in a single clinic: Exploring when and why they result in healthy pregnancies. Fertil Steril. 2019;111:280-293. DOI: 10.1016/j.fertnstert.2018.10.019.

56. Bolton H, Graham SJ, Van der Aa N, et al. Mouse model of chromosome mosaicism reveals lineage-specific depletion of aneuploid cells and normal developmental potential. Nat Commun. 2016;7:11165. DOI: 10.1038/ncomms11165.

57. Cystic Fibrosis Foundation. Available online at https://www.cff.org (last accessed June 3, 2019). 